\title{
Abordagem de aneurismas intracranianos em um hospital do baixo Amazonas
}

\author{
Intracranian aneurisms approach in a low Amazon hospital \\ Enfoque de aneurismos intracranos en un hospital de baja Amazonia
}

Ismael Henrique Azevedo De Alcântara ${ }^{1 *}$, Irilane De Alcântara Figueira ${ }^{1}$, Irineu Lopes de Alcântara Júnior $^{1}$, Luiz Henrique Nogueira ${ }^{1}$, Yasmin da Silva Nascimento ${ }^{1}$, Jociney José Pedroso Da Silva Junior ${ }^{1}$, Rudá Ávila Monteiro', David Isaac Serruya ${ }^{1}$, Miguel Rebouças de Sousa ${ }^{*}$, Ruan Davi Rebouças de Sousa1, lury José Rego Moura' e Juarez de Souza'.

\section{RESUMO}

Objetivo: Avaliar o perfil clínico dos pacientes diagnosticados com aneurisma intracraniano atendidos no Hospital Regional do Baixo Amazonas no período de janeiro de 2015 a dezembro de 2016. Métodos: Pesquisa transversal, quantitativa, descritiva e documental. Foram analisados os prontuários de 27 pacientes, dos quais foram retiradas informações sobre o sexo, idade, sinais e sintomas, fatores de risco para aneurisma intracraniano, localização, complicações da hemorragia subaracnóidea (HSA) e pós-operatória. Resultados: A maioria dos pacientes com ruptura de aneurisma cerebral era do sexo feminino e se encontravam na faixa etária de 41 a 60 anos; a cefaleia foi a queixa clínica inicial mais frequente; a HAS foi a comorbidade mais comum; a complicação mais encontrada após HSA foi a hidrocefalia; e a complicação pós-operatória mais frequente foi a formação de fístula liquórica. Conclusão: As mulheres são mais afetadas com aneurismas e estes podem causar diversas complicações graves, até o óbito.

Palavras-Chave: Aneurisma, Hemorragia Subaracnóidea, cefaleia.

\begin{abstract}
Objective: To evaluate the clinical profile of patients diagnosed with intracranial aneurysm seen at the Baixo Amazonas Regional Hospital from January 2015 to December 2016. Methods: cross-sectional, quantitative, descriptive and documentary research. The medical records of 27 patients were analyzed, from which information on gender, age, signs and symptoms, risk factors for intracranial aneurysm, location, complications of subarachnoid hemorrhage (SAH) and postoperative data were obtained. Results: Most patients with cerebral aneurysm rupture were female and were between 41 and 60 years old; headache was the most frequent initial clinical complaint; SAH was the most common comorbidity; most aneurysms were located in the posterior communicating artery; the most common complication after SAH was hydrocephalus and the most frequent postoperative complication was the formation of cerebrospinal fluid fistula. Conclusion: Women are more affected with aneurysms and these can cause several serious complications, even death.
\end{abstract}

Keywords: Aneurysm, Subarachnoid hemorrhage, Headache.

\section{RESUMEN}

Objetivo: evaluar el perfil clínico de pacientes diagnosticados con aneurisma intracraneal atendidos en el Hospital Regional Baixo Amazonas desde enero de 2015 hasta diciembre de 2016. Métodos: investigación transversal, cuantitativa, descriptiva y documental. Se analizaron los registros médicos de 27 pacientes, a

${ }^{1}$ Universidade do Estado do Pará - Campus XII. Santarém - PA. *E-mail: miguelreboucas1@gmail.com

SUBMETIDO EM: 9/2019

ACEITO EM: 10/2019

PUBLICADO EM: 11/2019

REAS/EJCH | Vol.Sup.36 | e1623 | DOI: https://doi.org/10.25248/reas.e1623.2019 Página 1 de 8 
partir de los cuales se obtuvo información sobre género, edad, signos y síntomas, factores de riesgo de aneurisma intracraneal, ubicación, complicaciones de hemorragia subaracnoidea (HSA) y datos postoperatorios. Resultados: la mayoría de los pacientes con rotura de aneurisma cerebral eran mujeres y tenían entre 41 y 60 años; el dolor de cabeza fue la queja clínica inicial más frecuente; SAH fue la comorbilidad más común; la mayoría de los aneurismas se ubicaron en la arteria comunicante posterior; La complicación más común después de la HSA fue la hidrocefalia y la complicación postoperatoria más frecuente fue la formación de una fístula de líquido cefalorraquídeo. Conclusión: las mujeres se ven más afectadas por los aneurismas y estos pueden causar varias complicaciones graves, incluso la muerte.

Palabras clave: Aneurisma, Hemorragia subaracnoidea, Dolor de cabeza.

\section{INTRODUÇÃO}

Hemorragia subaracnóidea (HSA) é o extravasamento de sangue dentro do espaço subaracnoide após algum evento vascular ou traumático. Cerca de $80 \%$ são causados por aneurismas cerebrais, localizados geralmente na bifurcação das artérias do polígono de Willis, mais frequentemente na circulação anterior (BRASIL NETO JP, 2013). Os aneurismas ocorrem devido a uma fragilidade da parede da artéria em forma de bolsa decorrente a diversos fatores.

Os aneurismas intracranianos podem ser divididos em seis tipos: sacular, fusiforme, arteriosclerótico, traumático, micótico e neoplásico, sendo os aneurismas saculares a maioria e localizam-se preferencialmente no polígono de Willis Os aneurismas ocorrem devido a uma fragilidade da parede da artéria em forma de bolsa decorrente a diversos fatores (MELO-SOUZA SE, 2008).

O aneurisma cerebral pode permanecer assintomático durante toda a vida do indivíduo, o que acontece na maioria das vezes. O pior cenário, no entanto, é o acontecimento da ruptura do aneurisma com hemorragia intracraniana, mais frequentemente no espaço subaracnóideo. Sabe-se que o risco de ruptura aumenta com o tamanho da lesão: por meio de estudos clínicos foi estabelecido como limite um tamanho de aneurisma de $7 \mathrm{~mm}$, abaixo do qual a sua ruptura seria muito rara em pacientes previamente sem sintoma de sangramento. Os pacientes com aneurismas de $7 \mathrm{~mm}$ ou mais têm risco de ruptura de 2,6 a 14,5\% em um período de cinco anos, dependendo da sua localização (CHAVES ML, et al., 2009).

As manifestações clínicas podem ser divididas em dois grupos: sinais e sintomas que compõem a classificação de Hunt-Hess e sinais e sintomas que não compõem a classificação de Hunt-Hess. No primeiro grupo, há cefaleia, sinais de irritação meníngea, alteração de nervos cranianos, déficits focais e alteração do nível de consciência. No segundo grupo, hemorragia ocular e dor lombar (BERTOLUCCI PHF, et al., 2011).

A prevalência do aneurisma intracraniano é desconhecida. Estima-se, no entanto, que 1 a $5 \%$ da população adulta apresente aneurismas cerebrais. A maioria dos aneurismas permanece assintomática até o dia do seu sangramento, o que ocorre na população em uma incidência estimada de 6 a 16 casos por 100.000 habitantes por ano (CHAVES ML, FINKELSZTEJN AFN, STEFANI MA, 2009).

O mais importante preditor do prognóstico da HSA é o estado clínico em que o paciente se apresenta na chegada do hospital. Inúmeros sistemas de classificação clínica da HSA foram elaborados ao longo dos anos, e a escala utilizada sistematicamente pelos neurocirurgiões para caracterização inicial da HSA, e que possui valor prognóstico, é a escala de Hunt-Hess. Porém, estudos mostraram que a variabilidade de classificação, entre diferentes observadores, usando essa escala é muito grande. Classificações baseadas na escala de Coma de Glasglow mostraram-se mais uniformes entre observadores distintos (MORAES NI, 2005).

Conhecer o perfil clínico dos pacientes com aneurisma intracraniano é essencial para o diagnóstico do aneurisma antes do seu sangramento principal, já que o rompimento aneurismático está associado a grande mortalidade e sequelas neurológicas. O prognóstico para os pacientes com ruptura de aneurismas sem tratamento é sombrio. Cerca de 60 a 70\% morrem até seis meses após a hemorragia (MELO-SOUZA SE, 2008). 
Não há um consenso geral entre os autores para tratar dos diferentes aspectos da HSA e os protocolos de manejo tem mudado consideravelmente ao longo do tempo, variando entre os diferentes centros e países. Recentemente, se tem adotado nas maiorias dos centros um protocolo mais uniforme, visando melhorar a evolução global da enfermidade e não somente nos casos selecionados para cirurgia. Os objetivos fundamentais para um correto tratamento da enfermidade são: 1. Diagnóstico precoce, 2. Prevenção de ressangramento, 3. Estabilização do paciente crítico em unidade de terapia intensiva e 4. Prevenção e tratamento agressivo da isquemia cerebral, especialmente nos casos em que se há ocluído o aneurisma (SOCIEDADE ESPANHOLA DE NEUROCIRURGIA, 2011).

O objetivo do presente estudo foi traçar o perfil clínico dos pacientes com aneurisma intracraniano atendidos no Hospital Regional do Baixo Amazonas, dando ênfase a informações como sexo, idade, manifestações clínicas que levaram o paciente a procurar atendimento médico, presença de comorbidades, localização do aneurisma e complicações decorrentes da hemorragia subaracnóidea e do procedimento cirúrgico.

\section{MÉTODOS}

O estudo tratou-se de uma pesquisa transversal, quantitativa, descritiva e documental, realizada no Hospital Regional do Baixo Amazonas (HRBA) em Santarém, um município da Região Oeste do Pará com população de 296.302 habitantes (IBGE, 2017). Nos anos de 2015 e 2016, 27 pacientes internaram para procedimento cirúrgico e clipagem de aneurisma intracraniano, dos quais foram retiradas informações sobre o sexo, idade, sinais e sintomas, fatores de risco para aneurisma intracraniano, localização dos aneurismas, complicações da hemorragia subaracnóidea e pós-operatória.

Foram incluídos todos os pacientes diagnosticados com aneurisma cerebral, havendo ou não outras patologias de origem cerebrovascular e foram excluídos todos os pacientes com prontuários preenchidos de maneira inadequada ou ilegível ou que por algum motivo não se foi possível obter acesso ao mesmo.

O estudo foi aprovado pelo Comitê de Ética em Pesquisa da Universidade do Estado do Pará, campus XII, com o CAAE: 67572017.9 .0000 .5168 e sendo os dados quantificados a partir da frequência absoluta e relativa, mediante utilização do programa Excel.

\section{RESULTADOS E DISCUSSÃO}

O sexo compõe um dos fatores de risco para formação e ruptura aneurismática, as hemorragias subaracnóideas (HSA) ocorrem com mais frequência entre os 40 e 60 anos de idade, sendo mais comum nas mulheres que nos homens (MAYER AS, et al., 2018). No presente estudo, a incidência de aneurismas intracranianos, em 66,6\% ( $n=18$ ) foi em mulheres e em 33,3\% ( $n=9)$ em homens, demostrando uma proporção de duas mulheres acometidas para cada homem. Queiroz JMVP (2010) afirma que a incidência de hemorragias subaracnóideas é maior em mulheres, relatando que dentre as causas (traumáticas, malformações, aneurisma), em torno de $75-80 \%$ seriam de etiologia aneurismáticas, segundo o mesmo trabalho, um específico é a pós-menopausa.

Ranski DBF (2005), na sua tese de doutorado sobre menopausa e fatores de riscos associados à doença cardiovascular, discute sobre a influência das alterações hormonais do pós-menopausa no aumento da incidência de doenças cérebro e cardiovasculares, assim o déficit estrogênico influencia a composição da parede arterial e a síntese de substâncias vasoativas, favorecendo a produção de colágeno e elastina e a proliferação de células musculares lisas induzidas por LDL colesterol, determinando mudanças da função do endotélio vascular, isto é, o hipoestrogenismo atua no sistema nervoso autônomo, provocando alterações vasomotoras e pressóricas que aumenta a resistência e endurecimento vascular e reduz sua complacência.

Sendo assim, os resultados corroboram com os dados obtidos por outros autores, ou seja, pode-se inferir que fatores hormonais (menopausa), podem servir como fatores que predispõe mais o sexo feminino em relação sexo masculino na formação e ruptura de aneurismas intracranianos. 
A idade é um importante fator de risco, havendo aumento na incidência de ruptura aneurismática com o aumento da idade. Esta ainda mantém relação com o sexo, visto que antes dos 50 anos, a ruptura desses aneurismas é mais comum nos homens, e, acima dessa idade, a tendência se inverte (MORAES NI, et al. 2005). Com relação a este fator, demonstra-se na Figura 1.

A maioria dos pacientes (48\%; $n=13)$ estavam na faixa etária de 41 a 60 anos, 29,8\% (n=8) tinham acima de 60 anos, $14,8 \%(n=4)$ entre $21-40$ anos e $7,4 \%(n=2)$ entre 0 e 20 anos, demonstrando um claro aumento na incidência de aneurisma intracraniano na $4^{2}$ e $5^{\mathrm{a}}$ décadas de vida, coincidentemente, sendo este o período da peri e pós menopausa feminina. Da mesma maneira que o presente trabalho, o estudo de Creôncio SCE, et al. (2015) encontraram uma maior incidência nas faixas e etárias de 41 a 50 anos, com $44 \%$ dos pacientes nessa categoria.

Figura 1 - Faixa etária de acometimento em anos.

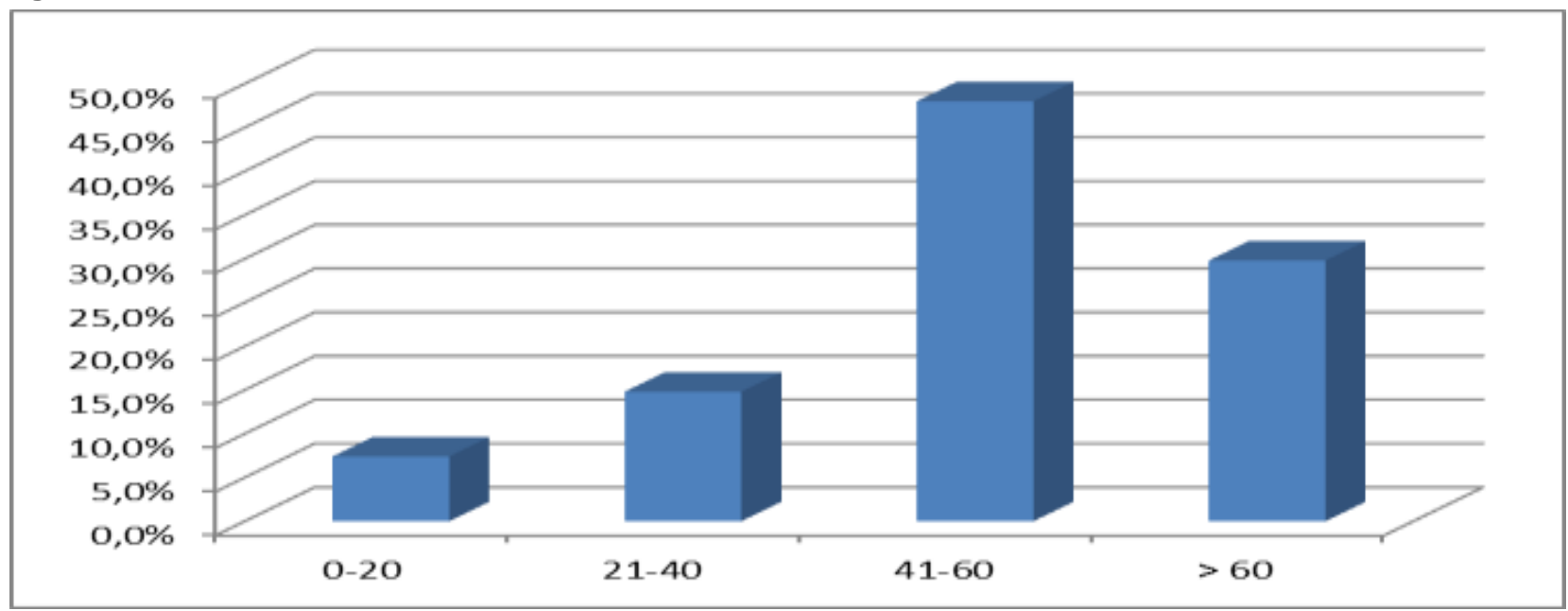

Fonte: Alcântara IHA, et al., 2018.

Em relação as manifestações clínicas relatadas pelos pacientes registrados no prontuário ao procurar atendimento médico (Figura 2), aproximadamente 56\% ( $n=15)$ dos pacientes relataram cefaleia, 30\% $(n=8)$ tiveram alteração do nível de consciência, $15 \%(n=4)$ tiveram rigidez de nuca e $15 \%$ náuseas e vômitos, sendo outros sintomas em menores quantidades.

Figura 2 - Manifestações Clínicas dos Aneurismas deste estudo.

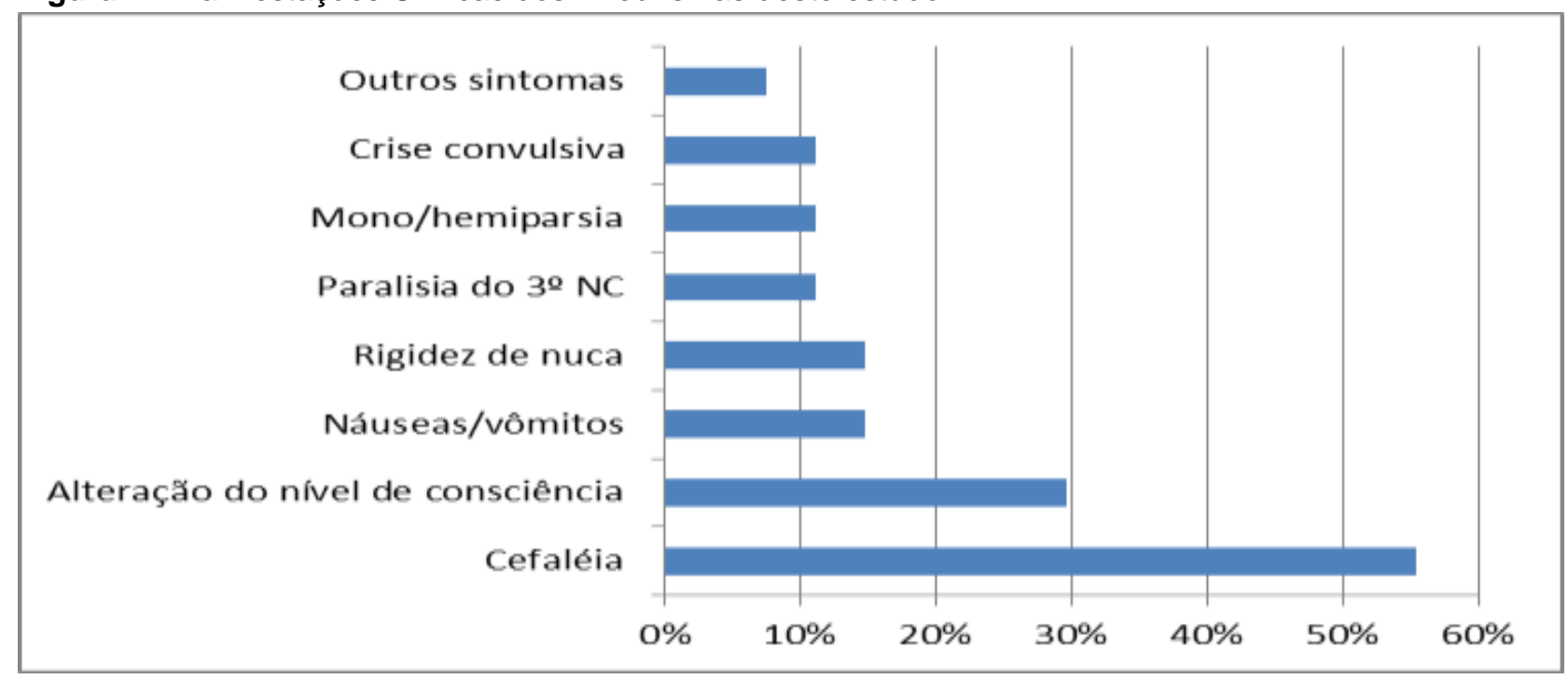

Fonte: Alcântara IHA, et al., 2018. 
A cefaleia é o sintoma mais comum em um quadro de hemorragia subaracnóidea, ocorrendo em $97 \%$ dos casos. Quanto à alteração de nervos cranianos, o sinal mais clássico é a alteração do III nervo craniano devido à compressão por aneurismas de comunicante posterior (BERTOLUCCI PHF et al., 2011).

Silva GC, et al. (2014), semelhante ao presente estudo, encontrou $48 \%(n=44)$ dos pacientes entre 31 e 50 anos e $42,9 \%$ ( $n=39)$ entre 51 e 85 anos, mostrando em seu trabalho uma maior tendência de aneurismas em idades mais tardias, associado a outros de fatores de risco da doença que, segundo o mesmo estudo, seriam a Hipertensão Arterial Sistêmica (HAS) e o tempo de tabagismo. Em resumo, a idade mais prevalente de aneurismas, bem como sua ruptura, estaria no período compreendido entre $4^{\mathrm{a}}$ e $5^{\mathrm{a}}$ décadas de vida, e alguns sinais e sintomas são mais comuns na ruptura ou na iminência de ruptura de um aneurisma intracraniano.

Silva GC, et al. (2014), estudando vítimas de hemorragia subaracnóidea não traumática, 84,6\% dos pacientes apresentaram cefaleia explosiva holocraniana e 19,8\% apresentaram crise convulsiva. Sinais e sintomas premonitórios são informados por 5 a $70 \%$ dos pacientes, aproximadamente metade dos indivíduos afetados buscam atendimento médico antes da hemorragia subaracnóidea principal e 16 a $60 \%$ desses pacientes são diagnosticados de maneira errada (QUEIROZ JMVP, 2010). Essa dificuldade do profissional médico em suspeitar de aneurisma intracraniano antes da ruptura aneurismática pode estar relacionada à inespecificidade dos sintomas e a possibilidade de vários outros diagnósticos diferenciais.

Os resultados das pesquisas citadas vão ao encontro do resultado obtido na presente pesquisa, demonstrando também a importância de relacionar os sinais e sintomas com fatores de risco como sexo, idade, presença de comorbidades, para um diagnóstico precoce de pacientes com aneurisma intracraniano antes da ruptura aneurismática principal, além da importância de se ter acesso aos meios diagnósticos como Angio-TAC e Angio-Ressonância na suspeita de aneurisma intracraniano.

Como demonstrado na figura 3, aproximadamente $64 \%(n=16)$ dos pacientes apresentavam hipertensão arterial sistêmica (HAS), 8\% ( $n=2)$ diabetes mellitus (DM), 8\% $(n=2)$ hemorragia subaracnóidea previa (HSA prévio) e $20 \%(n=5)$ apresentavam outras comorbidades como neuropatia periférica, obesidade, nefrolitíase, esteatohepatite e anemia falciforme. No estudo de Silva GC, et al. (2014), assim como o presente estudo, constataram que a HAS era o fator de risco mais prevalente, estando presente em $71,2 \%$ dos pacientes. Loureiro $A B$, et al. (2015) relatam em seu estudo como importantes fatores de risco a HAS, o uso de anticoncepcional oral (ACO), o abuso de substâncias como tabaco, cocaína e álcool, gestação, hipercolesterolemia e esforço físico. Os aneurismas adquiridos resultam de estresse hemodinâmico prolongado e concomitante a degeneração arterial nos pontos de saída dos principais ramos arteriais e bifurcações das maiores artérias cerebrais (LOUREIRO AB, et al., 2015).

Figura 3 - Comorbidades dos pacientes com aneurismas intracranianos.

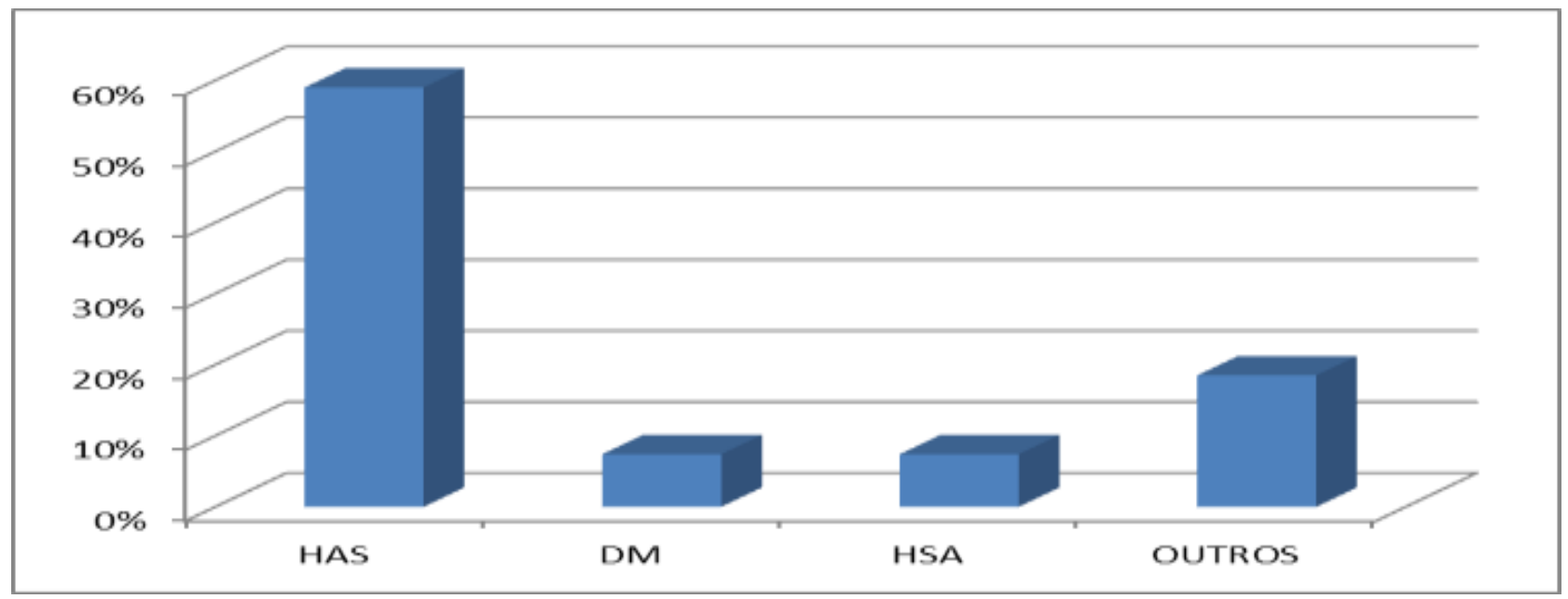

Fonte: Alcântara IHA, et al., 2018. 
A lesão endotelial, degenerescência da lâmina elástica interna e o afilamento da camada muscular lisa da média são sinais precoces da formação aneurismal. A hipertensão arterial afeta potencialmente essas três estruturas, podendo ser um fator predisponente da formação de aneurismas (QUEIROZ JMVP, 2010).

Raskin (2005) em seu trabalho cita pesquisas populacionais prospectivas americanas e europeias em mulheres entre 35 e 75 anos, destacando uma possível associação entre o déficit estrogênico, característico do climatério e pós-menopausa, e mecanismos reguladores da pressão arterial, considerando aquele como determinante de risco para hipertensão arterial. Sugere-se, entretanto, que a deficiência estrogênica esteja associada a outros fatores como obesidade, distribuição central da gordura e resistência insulínica. Os resultados encontrados no presente estudo seguem a mesma linha de outros estudos e da literatura, demonstrando a HAS como uma das principais comorbidades e fatores de risco presentes em pacientes com aneurisma intracraniano, e a necessidade do correto controle pressórico desses pacientes.

Como demonstrado acima, na figura 4, aproximadamente 15\% ( $n=4)$ apresentaram hidrocefalia, $7 \%(n=2)$ tiveram ressangramento, $7 \%$ vasoespasmo. Loureiro $A B$, et al. (2015) em seu trabalho relata que complicações como vasoespasmo, ressangramento, crise convulsivas, hidrocefalia e infecções podem levar os pacientes a óbito e causar grave comprometimento neurológico. Ele cita ainda um estudo feito com 3521 pacientes em que se observou que a maior causa de óbito e disfunções neurológicas severas foi 0 vasoespasmo com $13 \%$ dos pacientes, seguido de ressangramento com $7,5 \%$. Quanto à disfunção neurológica, a hidrocefalia representou $13 \%$ e as crises convulsivas $4,5 \%$.

Figura 4 - Complicações são mais frequentes após episódio de hemorragia subaracnóidea (HSA).

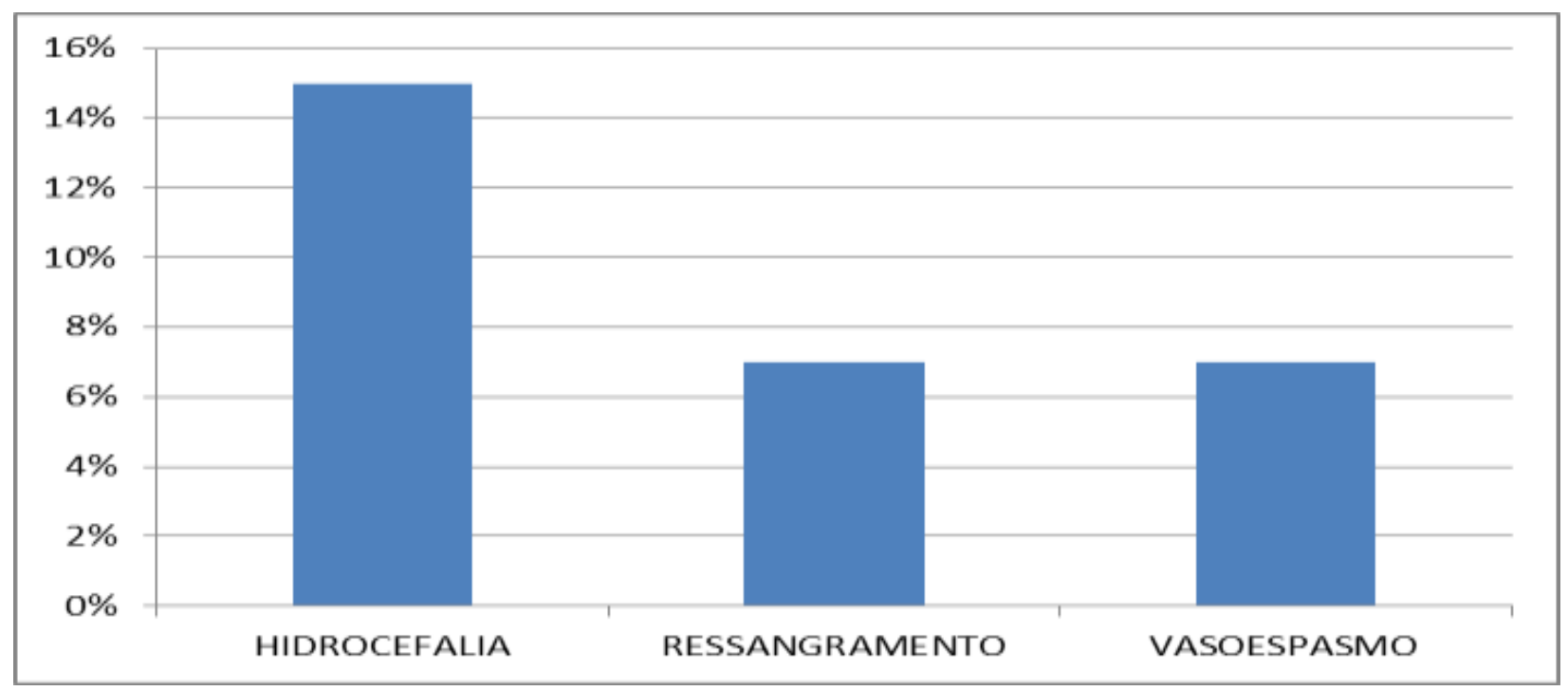

Fonte: Alcântara IHA, et al., 2018.

A HSA aneurismática, apesar de todos os avanços médicos e cirúrgicos, continua sendo uma doença com altos índices de mortalidade (40 a 50\%) e morbidade (MORAES NI, et al. 2005). Dos que sobrevivem até quatro meses após o sangramento inicial, $25 \%$ estão dependentes para realizar suas atividades diárias e mais de $80 \%$ tiveram redução da qualidade de vida. O tratamento dos pacientes com HSA deve dirigido para a prevenção do ressangramento, para prevenção ou tratamento do vasoespasmo e para a possível redução dos déficits neurológicos isquêmicos agudos (COLLI BO, et al., 1993).

Neste trabalho também foi verificado a presença de algumas complicações pós-cirúrgicas (figura 5). Chaves et al. (2009) coloca como complicações pós-operatórias os hematomas, fístulas liquóricas, meningites, infecções subcutâneas e de retalho ósseo. Nos pacientes submetidos ao procedimento cirúrgico no HRBA, $7,4 \%$ dos pacientes $(n=2)$ apresentaram formação de fístula liquórica, 3,7\% $(n=1)$ osteomielite e $3,7 \%(n=1)$ formação de hematoma extradural. Essas complicações podem ser responsáveis por aumento no tempo internação dos pacientes, que neste trabalho foi em média de 7 a 10 dias após abordagem cirúrgica. 
Figura 5 - Complicações pós-cirúrgicas.

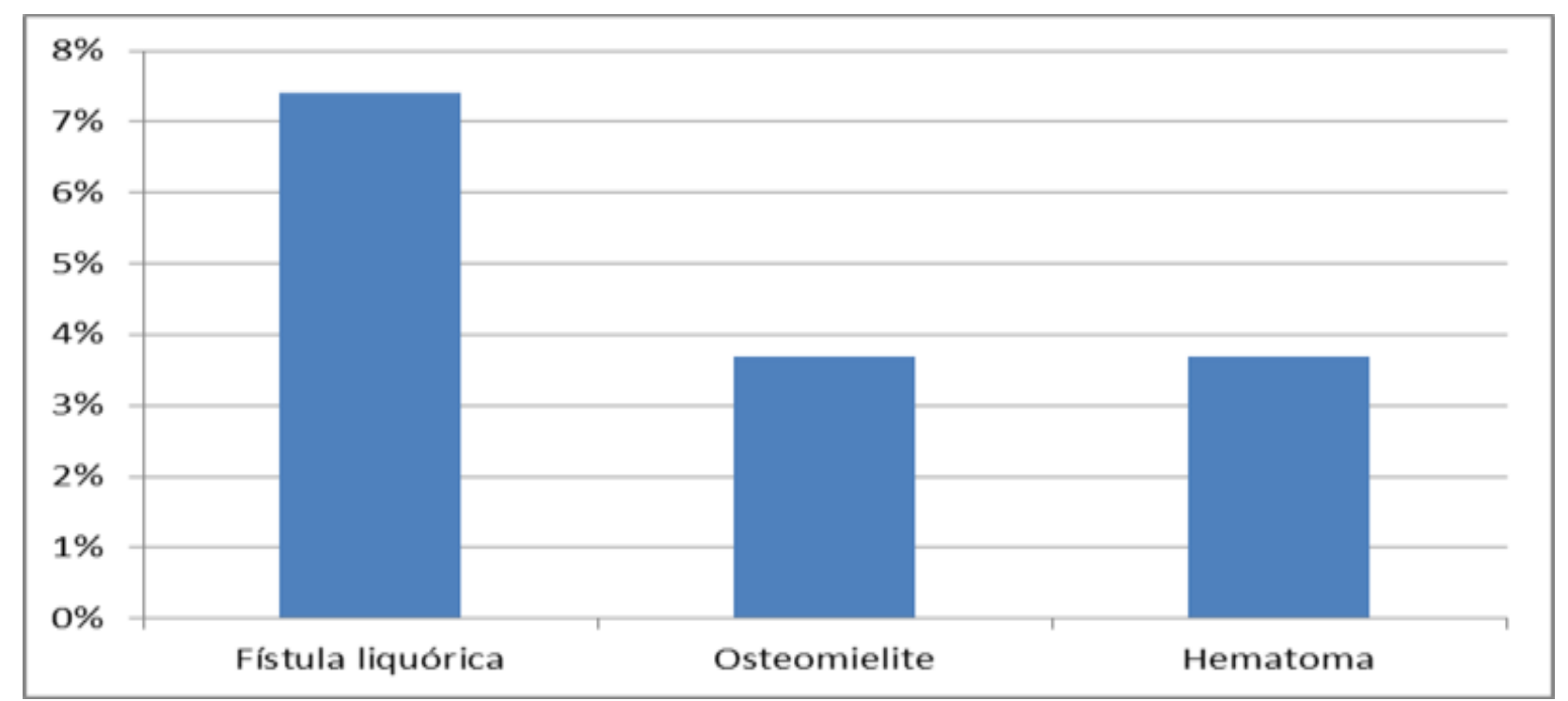

Fonte: Alcântara IHA, et al., 2018.

A maioria das HSA é causada por aneurismas rotos. Nessa situação, $40 \%$ dos pacientes vão a óbito, $30 \%$ sobrevivem com grandes sequelas e $30 \%$ retornam às atividades de forma funcional (BERTOLUCCI PHF et al., 2011). Percebe-se que a hemorragia subaracnóide é uma entidade catastrófica e suas complicações podem levar o paciente a um prognóstico sombrio, sendo geradora de sequelas físicas e ao óbito, verificandose assim, a importância do correto manejo de possíveis complicações.

\section{CONCLUSÃO}

Na presente pesquisa foi possível concluir que maioria dos pacientes com ruptura de aneurisma cerebral era do sexo feminino e se encontravam na faixa etária de 41 a 60 anos. Também se conclui que a cefaleia foi a queixa clínica inicial mais frequente, comumente designada pelos pacientes como a "pior dor de cabeça da vida", e a hipertensão arterial foi a comorbidade mais comumente encontrada nesses pacientes. Em relação à topografia; a complicação mais encontrada após a ruptura do aneurisma (hemorragia subaracnóidea) foi a hidrocefalia; e a complicação pós-operatória mais frequente foi a formação de fístula liquórica. Alguns fatores como falta de informações e reduzido número de participantes limitaram a realização do trabalho. Portanto, necessita-se de investimento em novas pesquisas acerca da problemática.

\section{REFERÊNCIAS}

1. AMERICAN HEART ASSOCIATION/AMERICAN STROKE ASSOCIATION. Guidelines for the Management of Aneurysmal Subarachnoid Hemorrhage. AHA Journal, $2012 . \quad$ Disponível: <http://stroke.ahajournals.org/content/strokeaha/43/6/1711.full.pdf> Acesso: 28 de novembro de 2016.

2. BERTOLUCCI PHF, et al. Guia de Neurologia. Barueri, SP. Manole, 2011. (Guias de medicina ambulatorial e hospitalar / editor Nestor Schor). p. 291-293.

3. BRASIL NETO JP; TAKAYANAGUI OM. Tratado de neurologia da Academia Brasileira de Neurologia. 1 ed. Rio de janeiro: Elsevier, 2013. p. 670-671.

4. CHAVES ML. FINKELSTEZN FN, STEFANI MA. Rotinas em neurologia e neurocirurgia. Porto Alegre: Artmed, 2009. p. 623-624, 626 e 627.

5. CHAVES ML, et al. Rotinas em neurologia e neurocirurgia. Porto Alegre: Artmed, 2009. p. 623-624, 626 e 627.

6. COLLI BO, et al. Tratamento cirúrgico dos aneurismas intracranianos - Comparação entre cirurgias precoce e tardia, Arq. Neuro-Psiquiatr. [Internet]. 1993 Mar [cited 2019 Sep 01] ; 51(1): 87-95.

7. CREÔNCIO SCE, et al. Análise de casos cirúrgicos em hemorragia subaracnóidea. Arquivos Brasileiros de Neurocirurgia,2015; 34: 7-14.

8. FREITAS JMM. Aneurisma de hiperfluxo próprio da artéria comunicante posterior decorrente de efeito hemodinâmico em paciente com oclusão da artéria subclávia tratado por angioplastia. Arquivo Neuropsiquiatria, 2005; 63 (3-A):693696. 
9. LOUREIRO, AB, et al. Evolução Funcional de Pacientes com Hemorragia Subaracnóide Aneurismática não Traumática, Revista brasileira de ciências da Saúde 19(2):123-128, 2015

10. MAYER AS, et al. Merritt - Tratado de Neurologia. 10 st ed. Rio de Janeiro: Guanabara Koogan, 2018. p. $222-223$.

11. MELO-SOUZA, SE. Tratamento das doenças neurológicas. 2 ed. Rio de Janeiro: Guanabara Koogan, 2008. p. 140141.

12. MORAES NI, et al. Tratado de clínica cirúrgica. São Paulo: Roca, 2005. p. 478.

13. MORAES NI. Tratado de clínica cirúrgica. São Paulo: Roca, 2005. p. 478.

14. QUEIROZ JMVP. Aneurismas Cerebrais - Qualidade de Vida e Estratégias de Prevenção a Adoptar. Dissertação (Mestrado em Gestão e Economia de Organizações de Saúde) - Faculdade de Economia, Universidade de Coimbra, Coimbra, 2010.

15. RASKIN DBF. Menopausa e fatores de risco associados à doença cardiovascular: Um estudo de coorte longitudinal. Tese (doutorado em Tocoginecologia) - Faculdade de medicina, Universidade Estadual de Campinas, Campinas, 2005.

16. SILVA BCG. Condutas no tratamento de aneurismas cerebrais: Concordâncias entre neurologistas. Monografia (Graduação em Medicina), Universidade Federal da Bahia, Salvador, 2013.

17. SILVA GC, et al.; Perfil clínico e terapêutico dos pacientes vítimas de hemorragia subaracnóidea não traumática no sistema único de saúde no município de Barbacena - MG, Rev Med Minas Gerais 2014; 24(3): $327-336$

18. SOCIEDADE ESPANHOLA DE NEUROCIRURGIA. Hemorragia subaracnóidea aneurismática: guia de tratamento del Grupo de Patologia Vascular de la Sociedad Espanõla de Neurocirugía. Revista de la Sociedad Espanõla de Neurocirugía. 22:(2), 2011.

19. TOWNSEND CM, et al. Sabiston - Tratado de Cirurgia. 19th ed. Rio de Janeiro: Elsevier, 2014. p. 1966. 